\title{
Editorial
}

\section{Obesidad y cáncer de próstata}

En referencia al artículo publicado en este número de Actas en las páginas 242-248

\author{
Manuel Martínez Sarmiento
}

Servicio Urología. Hospital La Fe. Valencia, España

$\mathrm{E}^{\mathrm{s}}$ ste comentario editorial surge tras la publicación en este número de la revista de un excelente artículo de revisión sobre la influencia que ejerce el tejido adiposo en el cáncer de próstata (CaP) que mediante la secreción de "adipoquinas", puede promover o prevenir el desarrollo del mismo e inducir su progresión. Sin embargo, a pesar de conocer los mecanismos fisiológicos que explican esta interrelación, persiste la controversia sobre el mayor riesgo de $\mathrm{CaP}$ en pacientes obesos, con resultados contradictorios en la literatura ${ }^{1,2}$. Una posible explicación a esta divergencia podría estar en la dificultad de detección del CaP en pacientes obesos, ya que los valores de PSA y las alteraciones del tacto rectal son menores en estos pacientes y el volumen prostático mayor ${ }^{1,2}$. Es decir, si los pacientes obesos tienen menores concentraciones séricas de PSA y alteraciones en el tacto rectal, tienen menos probabilidades de ser sometidos a biopsia prostática y de ser diagnosticados, por tanto, de CaP. Si además, los volúmenes prostáticos son mayores, la rentabilidad diagnóstica de la biopsia en estos pacientes, por consiguiente, es menor. Esto explica porque en un estudio de Freedland et al. ${ }^{3}$ los pacientes obesos sometidos a biopsia tenían menos probabilidad de tener un CaP, sin embargo tras ajustar los resultados en función del PSA y el volumen prostático, los obesos realmente tenían una mayor probabilidad de ser diagnosticados de $\mathrm{CaP}$ y de tener tumores de alto grado. Una actualización posterior de este grupo confirma estos resultados, ya que la obesidad, en un análisis crudo y ajustado por la edad, no se relaciona con el CaP, sin embargo cuando se ajusta en función del menor PSA y el mayor volu- men prostático, los obesos tienen casi el doble de riesgo de tener un $\mathrm{CaP}^{2}$. La trascendencia clínica de este hecho lleva a estos autores a sugerir bajar el valor de corte de PSA como criterio de biopsia en estos pacientes, como ocurre por ejemplo en los pacientes que toman inhibidores de la 5- $\alpha$-reductasa, así como aumentar el número de cilindros para minimizar los falsos negativos. Nuevos estudios deberán confirmar esta interrelación por sus implicaciones no sólo diagnosticas sino también terapéuticas, ya que los pacientes obesos con CaP son diagnosticados en general a edades más tempranas y con tumores de peor pronóstico ${ }^{4}$, un reto más en una patología, la obesidad, que constituye, en la actualidad, uno de los principales problemas de salud pública.

\section{REFERENCIAS}

1. Pruthi RS, Swords K, Schultz H, Carson CC 3rd, Wallen EM. The impact of obesity in the diagnosis of prostate cancer using a modern extended biopsy scheme. J Urol 2009;181(2):574-577.

2. Freedland SJ, Wen J, Wuerstle M, Shah A, Lai D, Moaleg B et al. Obesity is a significant risk factor for prostate cancer at the time of biopsy. Urology 2008;72(5):1102-1105.

3. Freedland SJ, Terris Mk, Platz EA, Presti JC Jr. Body mass index as a predictor of prostate cancer: development versus detection on biopsy. Urology 2005;66(1):108-113.

4. Motamedinia P, Korets R, Spencer BA, Benson MC, McKiernan JM. Body mass index trends and role of obesity in predicting outcome after radical prostatectomy. Urlogy 2008;72(5):11061110 .

Correspondencia autor: Dr. Manuel Martínez Sarmiento Servicio Urología. Hospital La Fe.

Avda. Campanar, 21 - 46009 Valencia, España.

Tel.: 963862700

E-mail autor: martinez_mansar@gva.es

Información artículo: Editorial

Trabajo recibido: febrero 2009 